\title{
ESTUDO DE ALTERNATIVAS DE COATING PARA TEMPERATURAS EXTREMAS DE BUSTLE GAS*
}

\begin{abstract}
Jean Philippe Santos Gherardi de Alencar ${ }^{1}$ Marcus Emrich ${ }^{1}$ Alei Domingues ${ }^{2}$

Resumo

O fenômeno de colagem é a formação de aglomerados de pelotas, denominados clusters, devido à sinterização do ferro gerado durante o processo de redução. $O$ resultado disto é a elevação da perda de carga nos reatores de redução direta. Tal fenômeno vem sendo estudado por vários pesquisadores, pois as práticas operacionais dos reatores têm priorizado o uso de temperaturas de bustle gas (BG) cada vez maiores, o que potencializa o aparecimento dos clusters. Uma alternativa para controlar a colagem é por meio de utilização de agentes de coating (AC's). Em relação às matérias primas utilizadas como $A C^{\prime}$ 's, as questões que surgem agora são as seguintes: Como se comportam os AC's em temperaturas muito elevadas de BG? Qual seria a maior temperatura possível de BG? A fim de começar a entender a melhor maneira de responder a estas questões foram realizados alguns testes de caráter exploratório e não convencionais para avaliar o comportamento de alguns materiais de coating a elevadas temperaturas de redução. Os resultados destes testes mostraram a possibilidade dos AC's funcionarem a temperaturas maiores que a de inicio do amolecimento da carga e que, nestas temperaturas, a sua granulometria pode ser parâmetro determinante para seu desempenho. 0 aprimoramento dos ensaios de simulação do processo de redução em reatores de redução direta é fundamental para responder as questões que se apresentam em relação ao tema.
\end{abstract}

Palavras-chave: Pelota; Redução direta; Colagem; Coating.

\section{STUDY OF ALTERNATIVE COATING TO HIGH TEMPERATURES OF BUSTLE GAS Abstract}

The clustering phenomenon consists of the sintered iron generated during the reduction process. The result of sintering could lead to the formation of strong clusters which disturb the gas flow inside the reactor. This phenomenon has been studied by many researchers because of client's movement in order to enhance the temperature of bustle gas to reach larger productivity. An alternative to control clustering is using coating agents (AC's). Above raw materials, the following questions arise now: What is the biggest possible temperature of BG? How behave the AC's in extreme temperatures? Some exploratory and unconventional tests were conducted to evaluate the behavior of some coating materials for high temperature reduction in order to begin to understand the best way to answer these questions. The results of these tests show that the AC's are able to work in higher temperatures than the charge's softening temperature and, at these temperatures, their particle size could be an extremely important characteristic. The intention is to enhance the evaluation tests and use them to definitively answer the questions that arose in relation to the problem.

Keywords: Pellet; Direct reduction; Clustering; Coating.

1 Engenheiro Metalurgista, Vale, Centro de Tecnologia de Ferrosos, Nova Lima, MG, Brasil.

2 Engenheiro Geólogo, Vale, Centro de Tecnologia de Ferrosos, Membro da ABM, Nova Lima, MG, Brasil.

* Contribuição técnica ao 44 Seminário de Redução de Minério de Ferro e Matérias-primas, 15ํ Simpósio Brasileiro de Minério de Ferro e $2^{\circ}$ Simpósio Brasileiro de Aglomeração de Minério de Ferro, 15 a 18 de setembro de 2014, Belo Horizonte, MG, Brasil. 


\section{INTRODUÇÃO}

Vários desenvolvimentos tecnológicos têm sido feitos no processo de redução direta a fim de aumentar a produtividade e diminuir os custos dos mesmos. Um deles foi o aumento da temperatura de Bustle Gas (BG). Com uma temperatura de BG maior a cinética de redução é favorecida e, consequentemente, a produtividade aumenta. GRISCOM et al [1] apresentam dados que apontam que artifícios como enriquecimento do gás natural, temperatura do gás redutor e adição de oxigênio, podem alcançar aumentos da ordem de $40-50 \%$ de produção quando se comparado às práticas originais dos reatores.

Além do aumento de produtividade, outras vantagens podem ser citadas como consequências do aumento de temperatura, que são elas: (i) aumento da metalização da carga; (ii) redução das perdas térmicas, já que o gás do reformador demanda menos tempo de resfriamento; (iii) elevação da resistência mecânica do DRI; e (iv) produção de DRI menos sensível a reoxidação. Em função disso, a tendência geral dos produtores de DRI é utilizar em suas operações as maiores temperaturas possíveis de BG.

O aumento de temperatura de BG, certamente, será limitado por algumas restrições de processo e matéria-prima. As limitações de matéria-prima, a princípio, estariam relacionadas ao fenômeno de colagem, o qual se pode definir como sendo a sinterização do ferro metálico gerado durante o processo de redução. O resultado dessa sinterização é a formação de aglomerados que, quando muito resistentes, são denominados "clusters", que prejudicam o escoamento gasoso e descida da carga ferrosa.

De acordo com a literatura [2-5], na faixa de temperatura em torno de $1000^{\circ} \mathrm{C}$ e mantidas constantes outros parâmetros de redução, a resistência dos "clusters" aumenta com a elevação da temperatura devido ao aumento da ductilidade e maleabilidade do material e aumento da densidade e espessura do ferro fibroso.

Num trabalho feito por Kortman et al [4] foi ressaltado que alguns ensaios de avaliação de colagem realizados tomando como base a norma ISO11256 e a temperatura $950^{\circ} \mathrm{C}$, tiveram que ser interrompidos a um grau de redução de apenas $84 \%$ devido ao alto diferencial de pressão do leito. Através dessa constatação, inferiu-se que a temperatura de redução dos testes para a pelota em questão estaria próxima da temperatura de amolecimento das mesmas e este seria o limite de temperatura de operação dos reatores.

É interessante destacar também outros trabalhos [6-8] que apontam para as diferenças de índices de colagem para diferentes condições de redução. $\mathrm{Na}$ temperatura de $950^{\circ} \mathrm{C}$ e composição igual ao teste do ISO11256, houve maior discriminação entre distintos agentes de coating no que diz respeito ao seu poder de inibição da formação de clusters e que os agentes mais ricos em "Mg" eram os mais eficazes [6]. Dentro desse contexto, o presente trabalho se propôs a realizar testes exploratórios e não convencionais visando um maior entendimento no que diz respeito a soluções de coating para condições de temperaturas em torno de $1050^{\circ} \mathrm{C}$.

\section{MATERIAIS E MÉTODOS}

\subsection{Materiais}

Foi utilizado um único tipo de pelota industrial $(\mathrm{P} 1)$ e quatro materiais de coating que, ao serem combinados e dosados, resultaram em seis diferentes tipos de

* Contribuição técnica ao $44^{\circ}$ Seminário de Redução de Minério de Ferro e Matérias-primas, 15오 Simpósio Brasileiro de Minério de Ferro e $2^{\circ}$ Simpósio Brasileiro de Aglomeração de Minério de Ferro, 15 a 18 de setembro de 2014, Belo Horizonte, MG, Brasil. 
pelotas recobertas para avaliação. Nas Tabelas 1 e 2, podem-se encontrar, respectivamente, as caracterizações química e física destes materiais de estudo.

Tabela 1 - Caracterização química dos insumos do estudo.

\begin{tabular}{cccccccccc}
\hline Materiais & $\mathrm{Fet}$ & $\mathrm{SiO}_{2}$ & $\mathrm{Al}_{2} \mathrm{O}_{3}$ & $\mathrm{CaO}$ & $\mathrm{MgO}$ & $\mathrm{Mn}$ & $\mathrm{P}$ & $\mathrm{TiO}_{2}$ & $\mathrm{PPC}$ \\
\hline Pelota P1 & 67.61 & 1.33 & 0.44 & 0.69 & 0.06 & 0.09 & 0.026 & 0.07 & 0.08 \\
Bauxita & 9.18 & 20.15 & 43.93 & 0.07 & 0.02 & 0.03 & 0.046 & 0.89 & 22.72 \\
Bent.+Cal.dolomítico & 3.28 & 14.73 & 4.63 & 21.90 & 15.51 & 0.21 & 0.025 & 0.41 & 34.81 \\
Serpentinito & 6.58 & 39.22 & 5.09 & 3.05 & 31.41 & 0.11 & 0.004 & 0.12 & 11.09 \\
\hline
\end{tabular}

Tabela 2 - Caracterização física dos insumos do estudo.

\begin{tabular}{cccccc}
\hline Amostra & P1 & Bauxita & Bentonita & $\begin{array}{c}\text { Cal. } \\
\text { Dolomítico }\end{array}$ & Serpentinito \\
\hline$\rho$ real $\left(\mathrm{g} / \mathrm{cm}^{3}\right)$ & 5,09 & 2,62 & 2,62 & 2,78 & 2,66 \\
$\rho$ aparente $\left(\mathrm{g} / \mathrm{cm}^{3}\right)$ & 2,18 & - & - & - & - \\
Granulometria $(\%-44 \mu \mathrm{m})$ & 0,00 & $75,01^{(1)}$ & $60,07^{(2)}$ & $67,19^{(2)}$ & $79,77[2]$ \\
Blaine $\left(\mathrm{cm}^{2} / \mathrm{g}\right)$ & - & 4930 & 2950 & 3780 & 11010 \\
Absorção água(\%) & - & - & 423 & - & - \\
Coloides(\%) & - & - & 69,18 & - & - \\
Inchamento(mL/2g) & - & - & 23 & - & - \\
\hline (1) Peneiramento a seco (Alpine). & & & & & \\
(2) Peneiramento a úmido & & & &
\end{tabular}

Em relação aos AC's, constatam-se algumas diferenças marcantes nas qualidades químicas, pode-se ver, por exemplo, que a Bauxita é um material rico em alumina, enquanto que o Serpentinito é rico em magnésio e a mistura Bentonita + Calcário Dolomítico em cálcio. Por outro lado, é notável também que o Serpentinito é o material mais fino e a mistura Bentonita + Calcário Dolomítico foi a que apresentou granulometria mais grossa.

\subsection{Métodos}

\subsubsection{Aplicação dos AC}

O recobrimento foi feito por meio de aspersão de solução aquosa dos agentes de coating sobre as pelotas dispostas em uma bandeja. Uma vez recobertas, as pelotas eram secas em estufa a $110^{\circ} \mathrm{C}$ por 2 horas e o percentual de material aderido foi determinado pela seguinte fórmula:

$$
I A=\left[(M f-M i)^{*} 100\right] / M_{M R}
$$

onde:

IA = índice de Aderência(\%);

$\mathrm{Mf}=$ massa de pelotas secas após aplicação de recobrimento(g);

$\mathrm{Mi}=$ massa de pelotas antes da aplicação do recobrimento $(\mathrm{g})$;

$\mathrm{M}_{\mathrm{MR}}=$ massa seca do material de recobrimento aplicado $(\mathrm{g})$.

\subsubsection{Redução das Amostras}

Todas as 06 (seis) amostras geradas no item anterior foram submetidas aos procedimentos de redução, conforme mostrado na Figura 1.

\footnotetext{
* Contribuição técnica ao 44 Seminário de Redução de Minério de Ferro e Matérias-primas, 15은 Simpósio Brasileiro de Minério de Ferro e $2^{\circ}$ Simpósio Brasileiro de Aglomeração de Minério de Ferro, 15 a 18 de setembro de 2014, Belo Horizonte, MG, Brasil.
} 

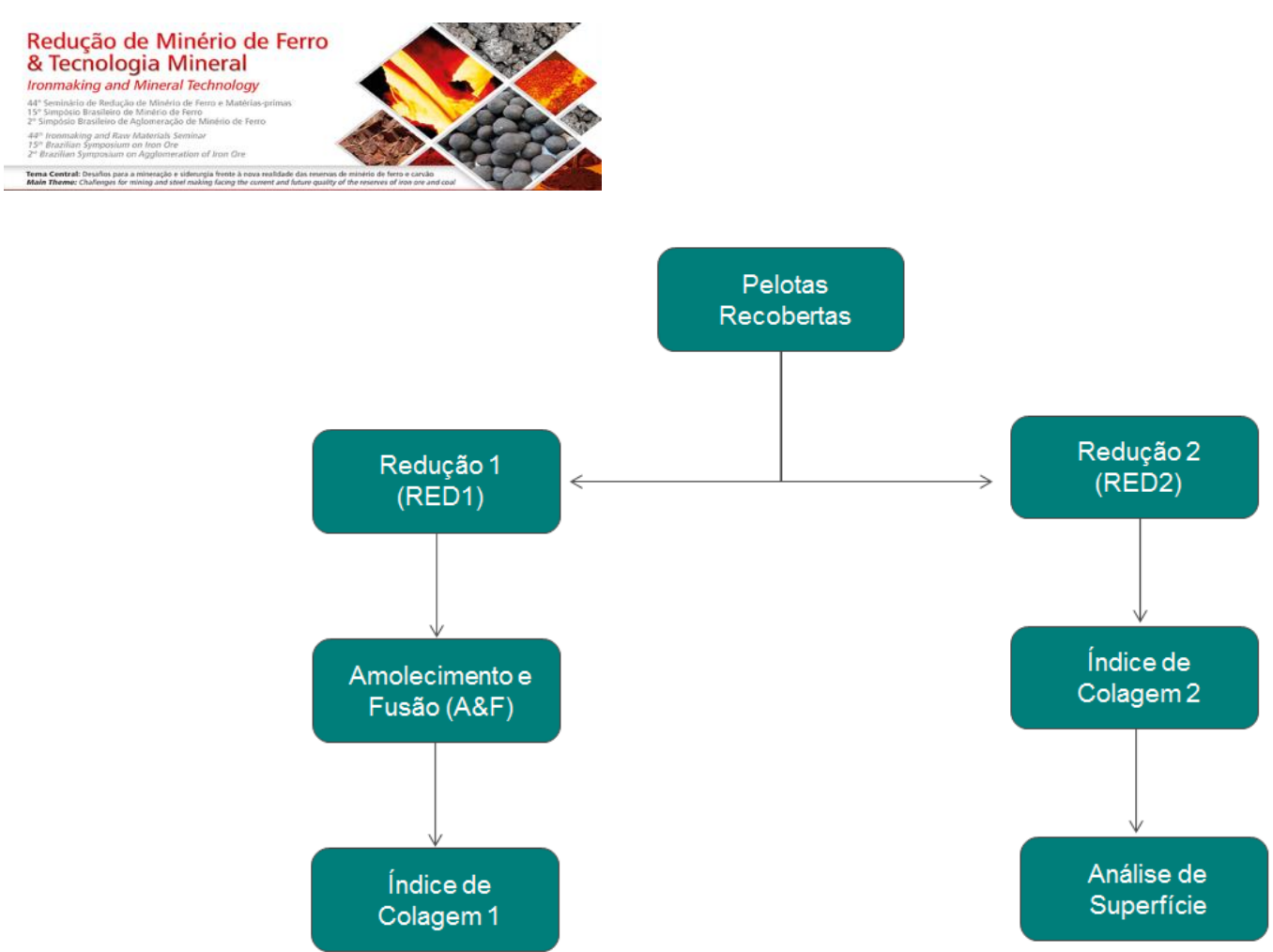

Figura 1 - Fluxograma de rotas de redução e avaliação pós-redução.

Basicamente foram criadas duas rotas de redução distintas que utilizaram equipamentos diferentes, mas com um propósito igual de avaliar os diferentes agentes de coating como inibidores da colagem em altas temperaturas.

$\mathrm{Na}$ rota RED1 as amostras foram submetidas a um ensaio de redução num forno RUL seguindo os parâmetros da norma ISO11256: Iron Ores Pellets - Determination of Clustering of Feedstock for Direct Reduction by Gas Reforming Process, mas sem aplicação de carga. Em seguida, o material gerado foi desagregado, quando necessário, e submetido a um ensaio num forno de amolecimento e fusão. A composição gasosa durante o ensaio foi $100 \%$ de $\mathrm{N}_{2}$, e utilizou-se uma carga sobre a amostra de $1,5 \mathrm{kgf} / \mathrm{cm}^{2}$, sendo o teste interrompido quando a pressão no interior do forno alcançou o valor de $200 \mathrm{mmH}_{2} \mathrm{O}$. Esse valor foi arbitrado como limite para identificação de início do amolecimento. Por fim, o material foi avaliado quanto ao seu índice de colagem de acordo com os procedimentos da norma ISO11256.

A rota RED2 utilizou o forno de redução RUL e, analogamente à rota RED1, baseouse na norma ISO11256, porém alterando a temperatura máxima do teste para $1050^{\circ} \mathrm{C}$. Posteriormente, o material foi avaliado quanto ao índice de colagem previsto na norma e caracterizado.

\subsubsection{Caracterização do Material Reduzido}

A Figura 2 mostra esquematicamente a estratégia usada para caracterização das amostras.

\footnotetext{
* Contribuição técnica ao 44 Seminário de Redução de Minério de Ferro e Matérias-primas, 15o Simpósio Brasileiro de Minério de Ferro e 2o Simpósio Brasileiro de Aglomeração de Minério de Ferro, 15 a 18 de setembro de 2014, Belo Horizonte, MG, Brasil.
} 


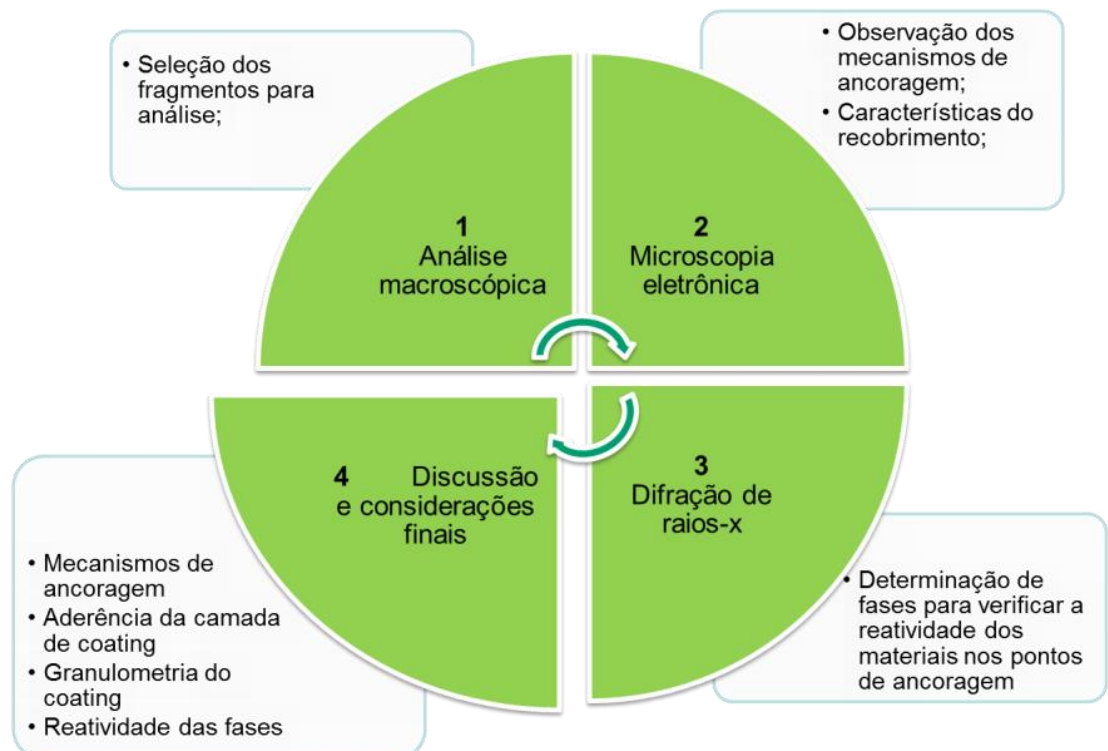

Figura 2 - Estratégia adotada para caracterização das amostras após RED2 e ensaio de colagem.

Os fragmentos selecionados para a caracterização das amostras são apresentados na Figura 3.

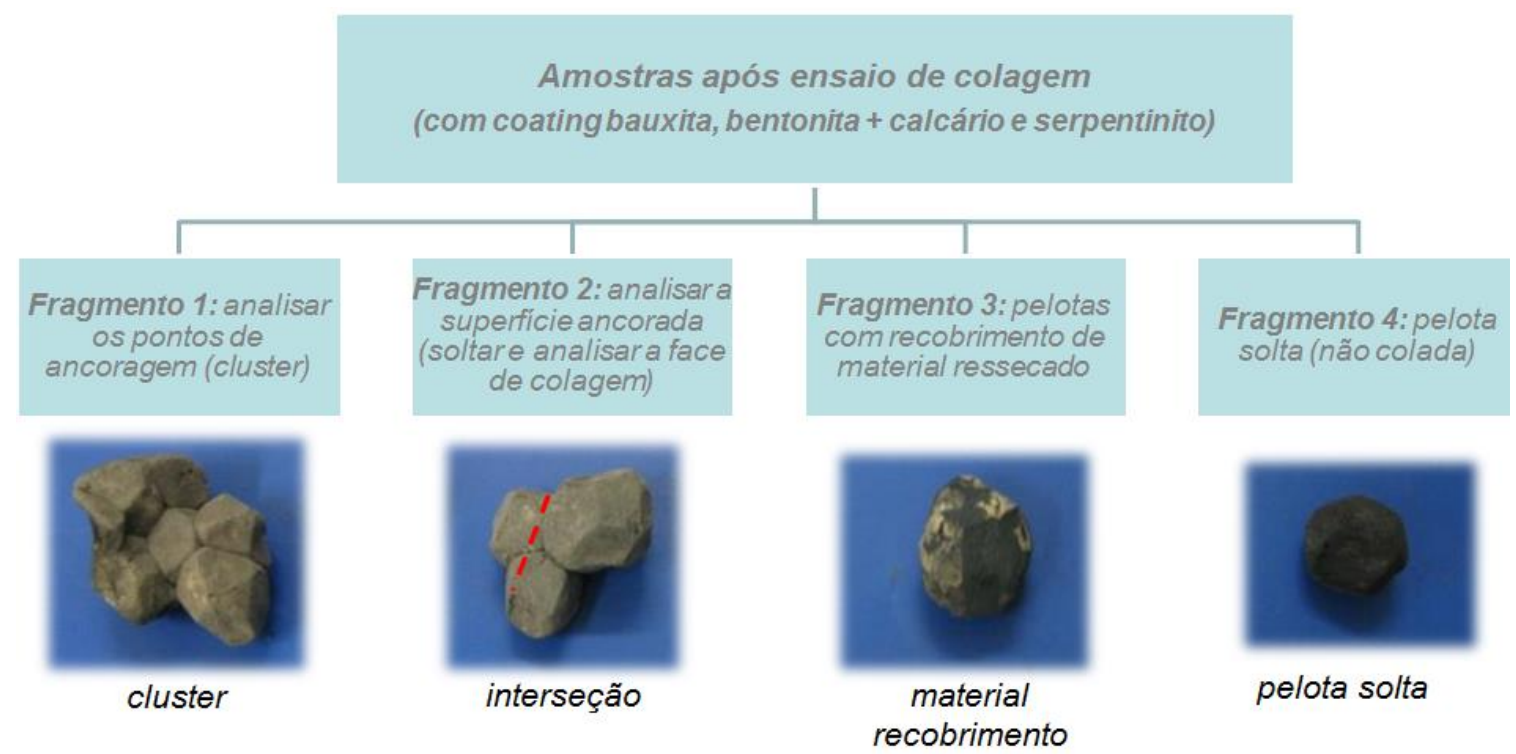

Figura 3 - Tipos de fragmentos selecionados para análise de imagens.

Para a análise de difração de raios- $\mathrm{X}$, as superfícies coladas (Fragmento 2) foram raspadas com um pincel de aço para que uma pequena quantidade de material fosse recolhida e analisada com o porta-amostra zero background, ideal para quantidades reduzidas de amostra, conforme mostra a Figura 4.

\footnotetext{
* Contribuição técnica ao $44^{\circ}$ Seminário de Redução de Minério de Ferro e Matérias-primas, 15은 Simpósio Brasileiro de Minério de Ferro e 2ํ Simpósio Brasileiro de Aglomeração de Minério de Ferro, 15 a 18 de setembro de 2014, Belo Horizonte, MG, Brasil.
} 

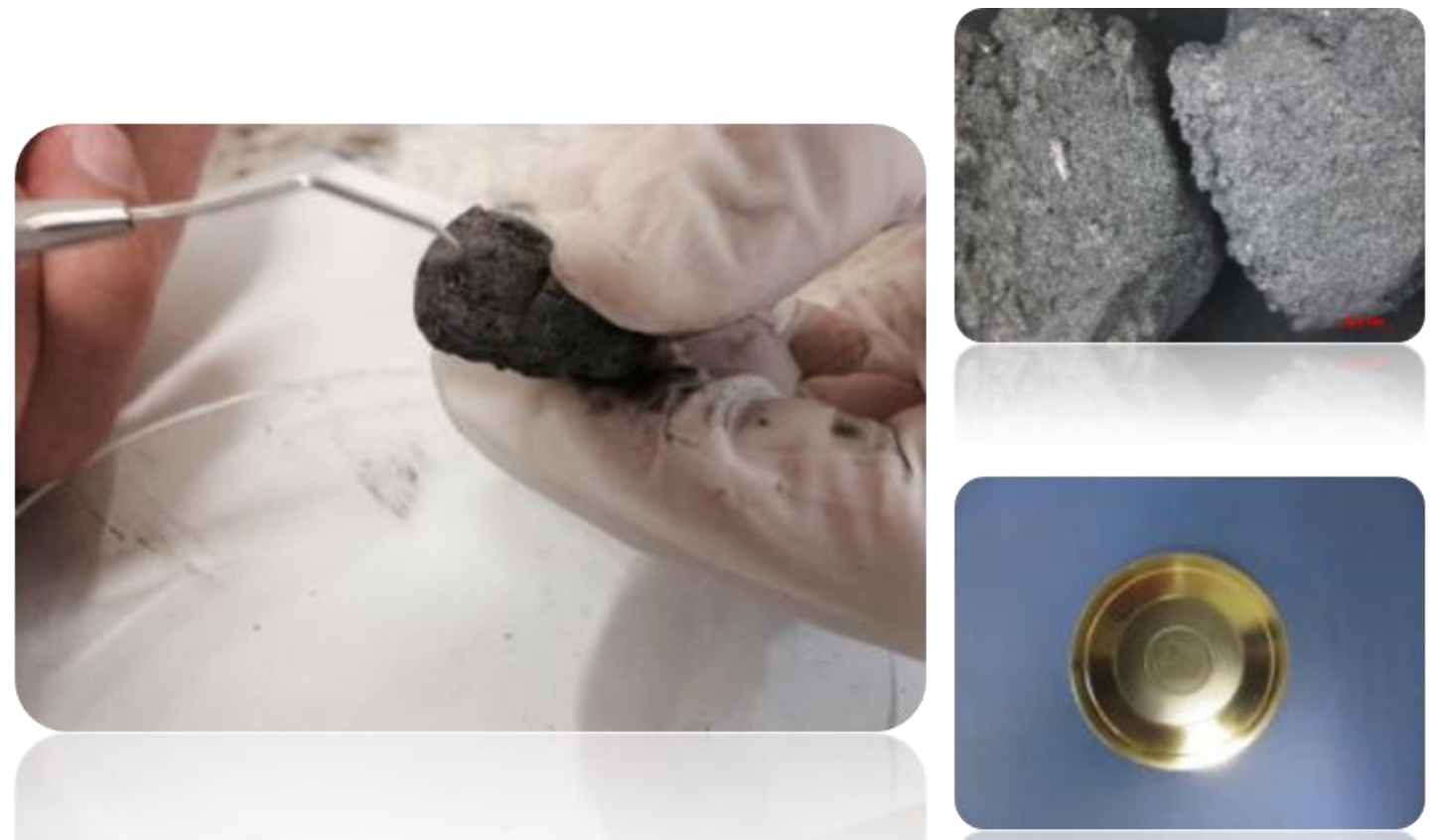

Figura 4 - Procedimento adotado para coleta de amostras para as análises de difração de raios-X das superfícies dos fragmentos.

\section{RESULTADOS E DISCUSSÃO}

\section{1 Índices de Aderência (IA's)}

O procedimento adotado para aplicação do material de recobrimento não foi assaz efetivo para maiores dosagens, já que o IA diminuiu à medida que aumentou a dosagem teórica, principalmente para a mistura de bentonita e calcário, a mais grossa. Apesar disto, as amostras que usaram um mesmo material de recobrimento obtiveram dois níveis distintos de dosagem efetiva, sendo um inferior e outro superior, como pode se ver na Tabela 3.

Tabela 3 - Dados das dosagens de cada material de coating.

\begin{tabular}{cccccccc}
\hline Amostra & $\begin{array}{c}\text { Material } \\
\text { recobrimento }\end{array}$ & $\begin{array}{c}\text { Dosagem } \\
\text { Teórica } \\
(\mathrm{g} / \mathrm{kg})\end{array}$ & $\begin{array}{c}\mathrm{Mi} \\
(\mathrm{g})\end{array}$ & $\begin{array}{c}\mathrm{Mf} \\
(\mathrm{g})\end{array}$ & $\begin{array}{c}\mathrm{M}_{\mathrm{MR}} \\
(\mathrm{g})\end{array}$ & $\mathrm{IA}(\%)$ & $\begin{array}{c}\text { Dosagem } \\
\text { Efetiva } \\
(\mathrm{g} / \mathrm{kg})\end{array}$ \\
\hline 1 & BAX & 3.0 & 8000 & 8023 & 24 & 95.8 & 2.9 \\
2 & BAX & 4.5 & 8000 & 8030 & 36 & 83.3 & 3.8 \\
3 & BEN+ CAD & 3.0 & 8000 & 8023 & 24 & 95.8 & 2.9 \\
4 & BEN + CAD & 4.5 & 8000 & 8029 & 36 & 80.6 & 3.6 \\
5 & SER & 3.0 & 8000 & 8022 & 24 & 91.7 & 2.8 \\
6 & SER & 4.5 & 8000 & 8030 & 36 & 83.3 & 3.8 \\
\hline
\end{tabular}

$\mathrm{Na}$ caracterização dos fragmentos, observou-se que, dentre as três amostras analisadas, a amostra recoberta com serpentinito (material mais fino) foi a que apresentou maior extensão de área recoberta, a qual foi avaliada mediante análise de imagens no microscópio ótico (Figura 5).

\footnotetext{
* Contribuição técnica ao 44 Seminário de Redução de Minério de Ferro e Matérias-primas, 15ํ Simpósio Brasileiro de Minério de Ferro e $2^{\circ}$ Simpósio Brasileiro de Aglomeração de Minério de Ferro, 15 a 18 de setembro de 2014, Belo Horizonte, MG, Brasil.
} 


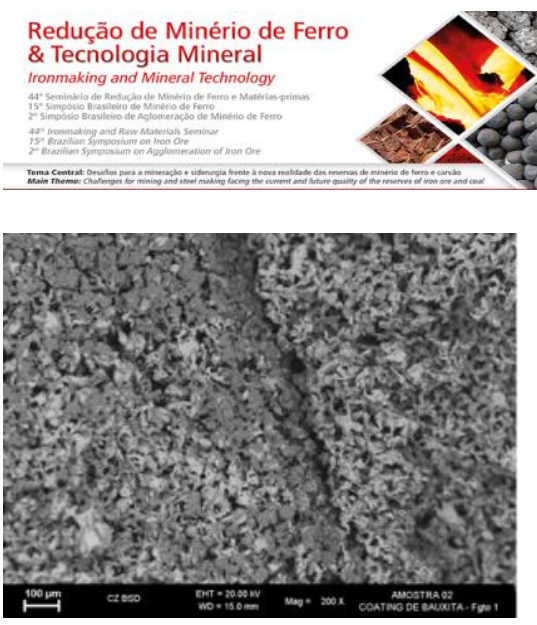

(a) Bauxita

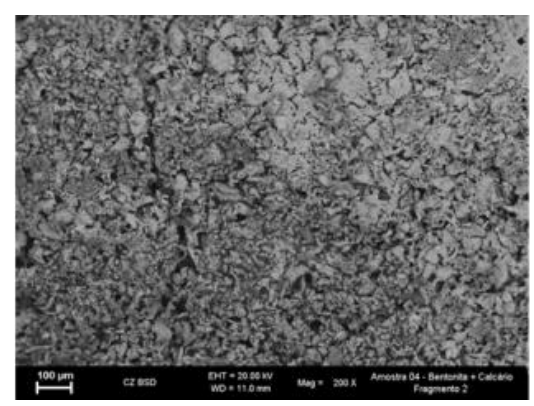

(b) Calcário + Bentonita

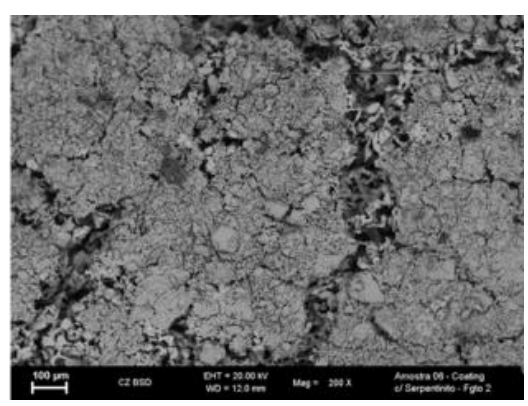

(c) Serpentinito

Figura 5 - Aderência da camada de AC.

\section{2 Índices de Colagem (IC's)}

Através da rota RED1 foram obtidas duas variáveis resposta no ensaio, que são: (i) Ts200, temperatura na qual a pressão no interior do forno foi de $200 \mathrm{mmH}_{2} \mathrm{O}$; (ii) Índice de colagem em \%, mesma relação sugerida na norma ISO11256. Enquanto isso, os materiais que foram submetidos à rota RED2 apresentaram o índice em \%, também seguindo a fórmula de cálculo da norma ISO11256. Os testes realizados seguindo a rota RED1 foram realizados uma única vez ao passo que os testes da rota RED2 seguiram o critério de aceitação da norma ISO11256 cujo número máximo de ensaios podia chegar a 4 (quatro). Na Tabela 4 são apresentados os dados obtidos após os ensaios de redução em função do tipo e quantidade de AC's utilizados.

Tabela 4 - Dados dos resultados de redução e índice de colagem.

\begin{tabular}{ccccccc}
\hline Amostra & Material & Dosagem & \multicolumn{2}{c}{ RED1 } & \multicolumn{2}{c}{ RED2 } \\
recobrimento & Efetiva $(\mathrm{g} / \mathrm{Kg})$ & IC $(\%)$ & $\mathrm{T}_{\text {s200 }}\left({ }^{\circ} \mathrm{C}\right)$ & IC $(\%)$ & $\mathrm{T}_{\max }$ \\
\hline 1 & BAX & 2.9 & 99.5 & 1153 & 89.5 & 1050 \\
3 & BEN + CAD & 2.9 & 99.8 & 1177 & 70.0 & 1050 \\
5 & SERP & 2.8 & 99.4 & 1144 & 25.4 & 1050 \\
2 & BAX & 3.8 & 97.8 & 985 & 80.4 & 1050 \\
4 & BEN + CAD & 3.6 & 93.4 & 930 & 49.3 & 1050 \\
6 & SERP & 3.8 & 97.3 & 1021 & 57.9 & 1050 \\
\hline
\end{tabular}

De maneira geral, os índices de colagem (IC) das amostras RED1, pós-redução, foram superiores aos índices obtidos com a RED2. Isto pode ser função do maior tempo de exposição das amostras RED1 a altas temperaturas (após serem reduzidas até $850^{\circ} \mathrm{C}$ no RUL, tais amostras eram desagregadas e posteriormente eram submetidas a um novo aquecimento, desta vez no forno de A\&F até a temperatura Ts200). Ainda no que diz respeito aos testes RED1 é preciso destacar o seguinte:

- A forte relação existente entre o IC e a temperatura máxima alcançada no teste, independente do tipo e quantidade de AC utilizado, conforme Figura 6.

- A redução do Ts200 com aumento da quantidade de AC utilizado, sugerindo reatividade do $A C$ com o seu substrato. $O$ material no qual este efeito foi menos pronunciado foi o serpentinito.

\footnotetext{
* Contribuição técnica ao 44ํㅗㄴ Seminário de Redução de Minério de Ferro e Matérias-primas, 15ㅇ Simpósio Brasileiro de Minério de Ferro e 2o Simpósio Brasileiro de Aglomeração de Minério de Ferro, 15 a 18 de setembro de 2014, Belo Horizonte, MG, Brasil.
} 
A menor reatividade do serpentinito com o substrato foi confirmada a partir da caracterização das superfícies dos fragmentos por difração de raios-X (Figura 7). Neste caso, formaram-se somente silicatos que não contém Fe após redução.

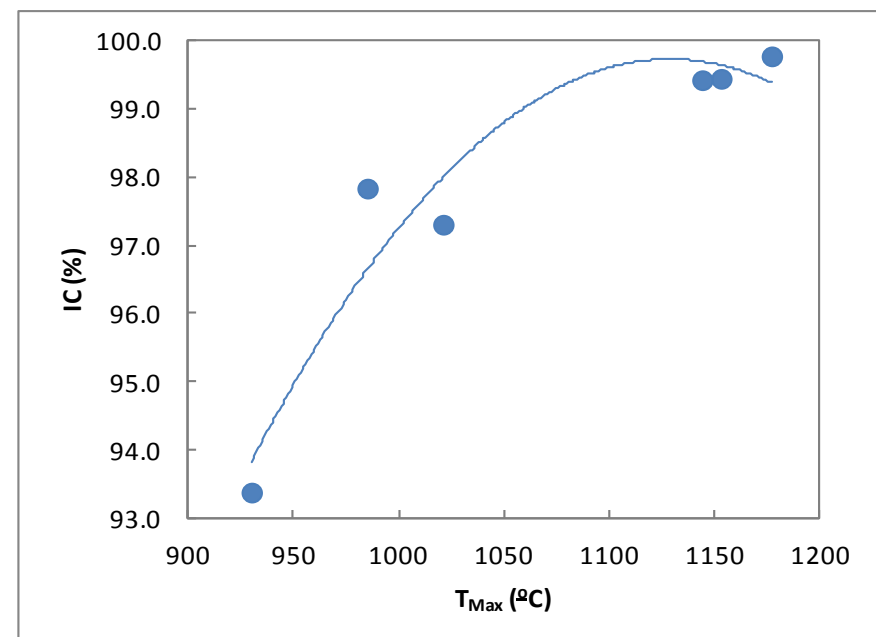

Figura 6 - Correlação entre o índice de colagem e a temperatura máxima alcançada no teste RED1.
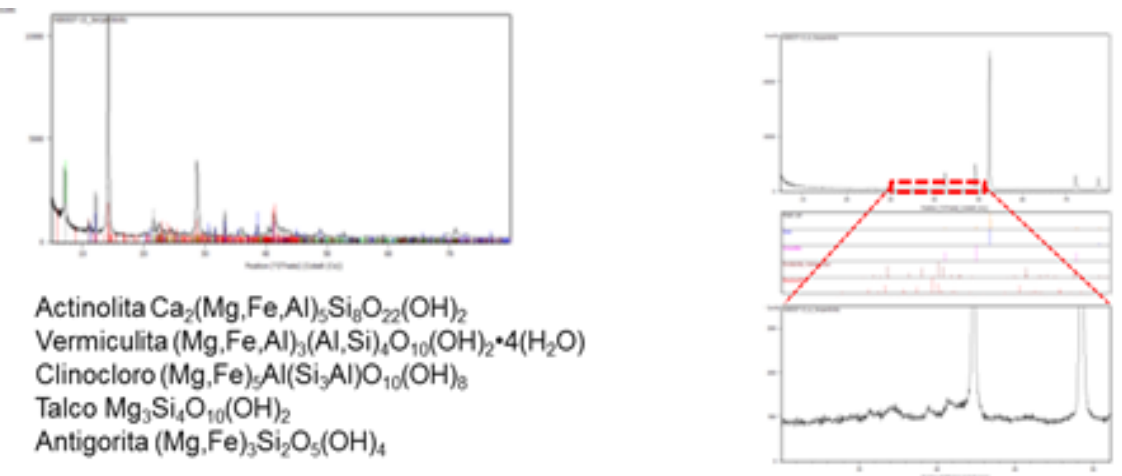

\author{
Ferro metálico: $\alpha-F e$ \\ Wustita: $\mathrm{FeO}$ \\ Silicato: $\mathrm{Mg}_{2} \mathrm{SiO}_{4}$ \\ Silicato: $\mathrm{CaMgSiO}_{4}$
}

Figura 7 - Difração de raios-x do serpentinito puro (à esquerda) e do pó extraído do material pós- redução.

Por outro lado, o índice de colagem dos produtos da RED2 foi dependente do tipo e quantidade do agente de recobrimento e, ao que parece, o desempenho deles dependeu da temperatura de amolecimento da carga (Ts200). Para Tred $<T s 200$, o material de melhor desempenho foi o Serpetinito, o menos reativo, e quando Tred>Ts200, o melhor desempenho foi o da mistura Bentonita + Calcário Dolomítico, que era a mais grossa. Percebe-se também, como mostra a Figura 8, que o Serpentinito foi o único material que ao se aumentar a dosagem de coating teve um índice de colagem superior. Tal fato pode estar relacionado a um segundo mecanismo de formação de aglomerados, a partir de reações no próprio material de recobrimento proporcionando a formação de pontes entre eles.

\footnotetext{
* Contribuição técnica ao 44ํㅗㄴ Seminário de Redução de Minério de Ferro e Matérias-primas, 15ํ Simpósio Brasileiro de Minério de Ferro e $2^{\circ}$ Simpósio Brasileiro de Aglomeração de Minério de Ferro, 15 a 18 de setembro de 2014, Belo Horizonte, MG, Brasil.
} 


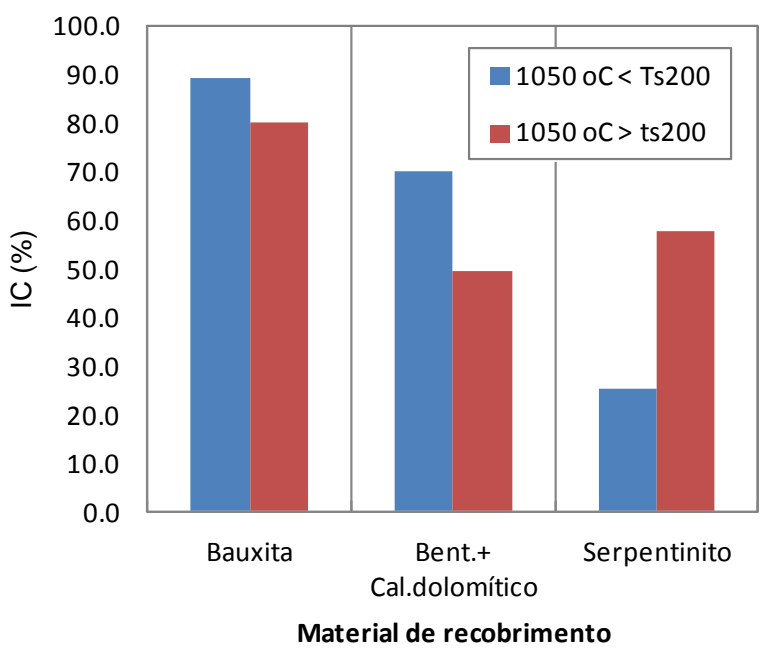

Figura 8 - Comparativo entre os materiais de coating e a temperatura de redução.

Um efeito análogo da granulometria e aumento de dosagem do AC sobre o IC a mais altas temperaturas foi também observado por Pereira [6], destaque na Figura 9, mas nenhuma hipótese foi formulada para explicá-lo.

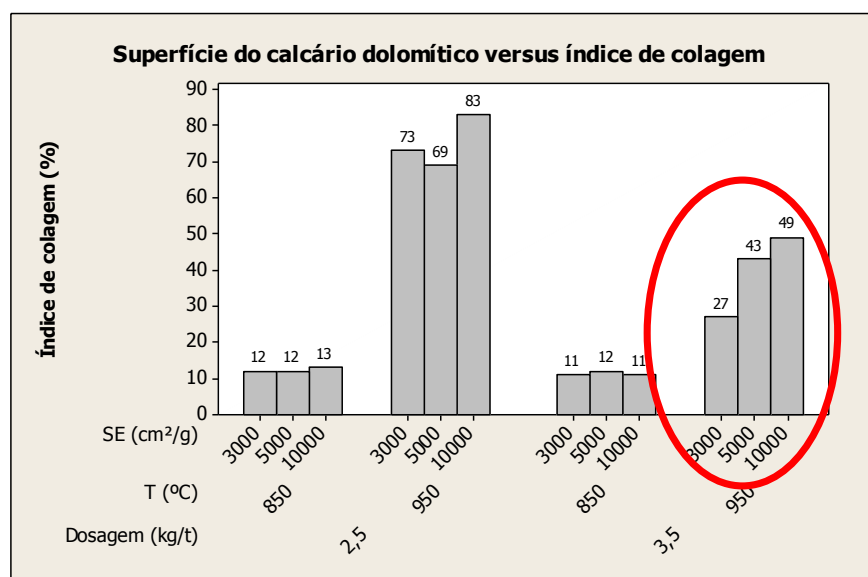

Figura 9 - Efeito da superfície do calcário no índice de colagem em diferentes temperaturas e dosagens [6].

\subsection{Caracterização do Material Reduzido}

As análises de caracterização foram realizadas conforme fluxograma anteriormente apresentado. Dentre as constatações feitas, é pertinente ressaltar que:

- Os fragmentos do tipo 3 confirmaram que o material ressecado é composto pelo próprio agente de coating. Foi contundente a percepção de que nestas regiões a granulometria do coating era grosseira.

- Nas áreas em que foram encontradas essas películas ressecadas não havia pontos de ancoragem, o que reforça que a formação dessas camadas de coating com granulometria grosseira são eficazes em evitar a colagem em mais altas temperaturas.

\footnotetext{
* Contribuição técnica ao $44^{\circ}$ Seminário de Redução de Minério de Ferro e Matérias-primas, 15ํ Simpósio Brasileiro de Minério de Ferro e $2^{\circ}$ Simpósio Brasileiro de Aglomeração de Minério de Ferro, 15 a 18 de setembro de 2014, Belo Horizonte, MG, Brasil.
} 

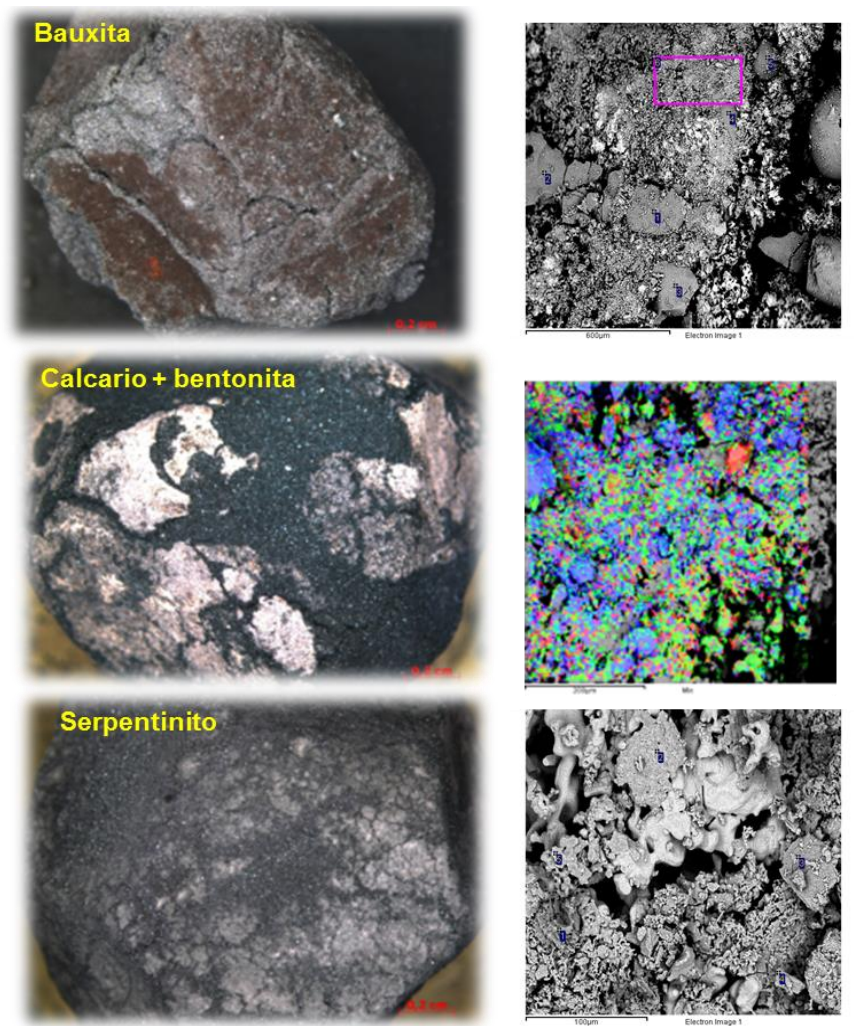

Figura 10 - Efeitos da granulometria do AC's sobre o fenômeno de colagem a altas temperaturas.

- Através da análise do fragmento tipo 1 do Serpentinito, percebe-se que a união entre as pelotas se deu não só pela ancoragem do ferro fibroso como também pela adesão de superfícies com material de recobrimento, todavia em menor frequência. Tal circunstância corrobora a justificativa vista nos testes do RED2 desse material, onde ao se aumentar a dosagem o índice de colagem também se elevou.

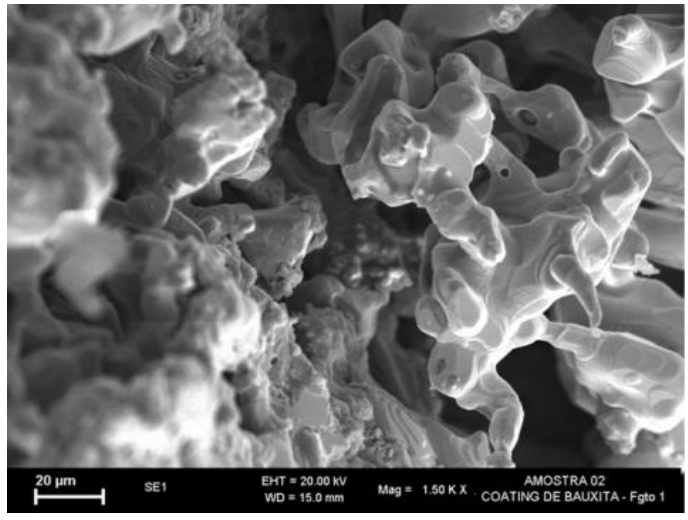

(a)

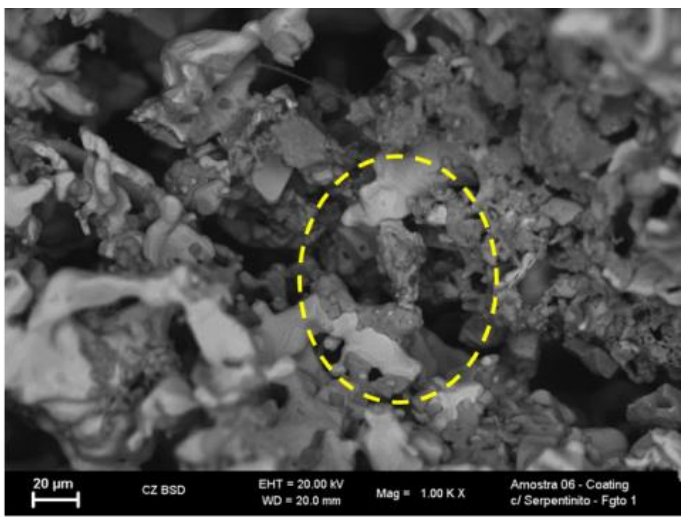

(b)

Figura 11 - (a) Ancoragem por pontes de ferro e (b) Ancoragem em superfícies recobertas.

\section{CONCLUSÕES}

A experiência de se avaliar o efeito de diferentes agentes de coating convencionais em elevadas temperaturas, utilizando equipamentos como o forno RUL e de A\&F, foi feita com êxito. Os principais pontos que merecem destaque nesse trabalho são:

\footnotetext{
* Contribuição técnica ao 44 Seminário de Redução de Minério de Ferro e Matérias-primas, $15^{\circ}$ Simpósio Brasileiro de Minério de Ferro e $2^{\circ}$ Simpósio Brasileiro de Aglomeração de Minério de Ferro, 15 a 18 de setembro de 2014, Belo Horizonte, MG, Brasil.
} 
- A partir dos resultados do forno de A\&F foi possível ter uma primeira impressão sobre o limite de amolecimento das cargas reduzidas. A maior temperatura de amolecimento obtida com os agentes de coating avaliados foi de $1177^{\circ} \mathrm{C}$. Esta temperatura pode ser uma estimativa inicial para balizar 0 alcance máximo da temperatura de bustle gas (BG).

- A quantidade de material de recobrimento aplicada tem influência sobre a temperatura de amolecimento da carga, ela diminuiu à medida que se aumentou a dosagem de recobrimento. Este efeito foi menor para os elementos com mais $\mathrm{MgO}$, ou menos reativos.

- Mesmo em temperaturas de redução acima do ponto de amolecimento da carga há possibilidades de se minimizar o fenômeno de colagem a partir do uso de agentes de coating. Todavia, os requisitos de reatividade $e$ granulometria dos mesmos devem ser pontos de atenção. Materiais menos reativos e mais grosseiros foram os de melhor desempenho.

- O aprimoramento das técnicas utilizadas neste trabalho, bem como a ampliação dos tipos e granulometrias dos agentes de coating, são fundamentais para a identificação das melhores soluções para o uso de altas temperaturas de BG.

\section{REFERÊNCIAS}

1 Griscom FN, Metius GE, Kopfle JT., Ironmaking Technology for the New Millenium. Direct from Midrex, 2nd Quarter, 2000. p.3-6.

2 Narita K, Kaneko D, Kimura Y. Study on clustering and its prevention in the SF for DR process; 97th ISIJ Meeting ; April 1979.

3 Wong PLM, Kim MJ, Kim HS, Choi CH. Sticking behavior in DR of iron ore; Ironmaking and Steelmaking; 1999, 26(1).

4 Kortman HA, Ritz VJ, Munnik B. Improvement of DR pellets related to sticking and process by application of specifically adjusted coating minerals, SGA/AKZO Nobel Chemicals.

5 Sterneland J, Jonsson PO. The use of coated pelllets in optimising the BF operation; ISIJ International, 2003; 43 (1).

6 Pereira JG. Avaliação da utilização de diferentes materiais para diminuição da tendência de colagem de pelotas durante o processo de redução direta; Dissertação de Mestrado; UFMG, 2012.

7 Lopes FS. Estudo do Fenômeno de Colagem das Pelotas Samarco Durante o Processo de Redução Direta. Ouro Preto: Universidade Federal de Ouro Preto, 2004. 136p. (Dissertação, Mestrado em Engenharia de Materiais).

8 Kobayashi M, Gudenau HW, Burchard WG, Schaefer HC. Fibrous Growth of Iron Precipitates during Reduction of iron Ores by CO Gas. ISIJ International, 1985, v. 71, n. 9, p.1102-1109.

\footnotetext{
* Contribuição técnica ao 44ํ Seminário de Redução de Minério de Ferro e Matérias-primas, 15ํ Simpósio Brasileiro de Minério de Ferro e $2^{\circ}$ Simpósio Brasileiro de Aglomeração de Minério de Ferro, 15 a 18 de setembro de 2014, Belo Horizonte, MG, Brasil.
} 